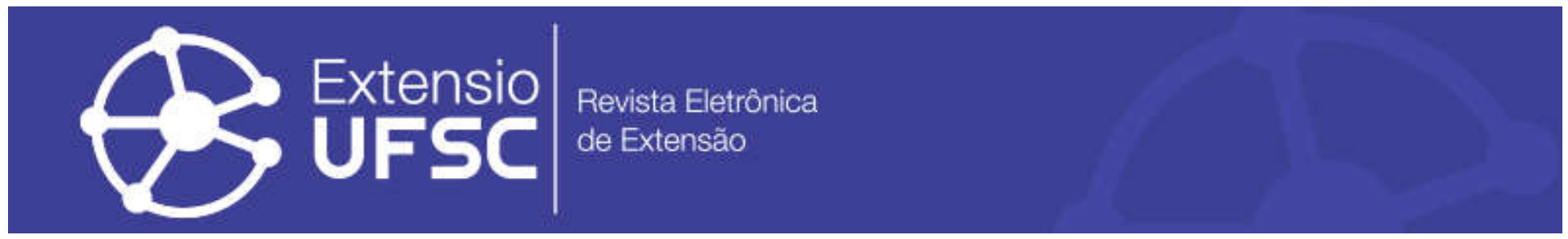

\title{
TRATAMENTO DE CRIANÇAS COM TRANSTORNOS DOS SONS DA FALA GRAVES OU PERSISTENTES POR MEIO DA ULTRASSONOGRAFIA DE LÍNGUA
}

\author{
Aline Mara de Oliveira \\ Universidade Federal de Santa Catarina \\ aline.mara.oliveira@ufsc.br \\ Patrícia Haas \\ Universidade Federal de Santa Catarina \\ haaspatricia37@gmail.com
}

\author{
Luciane Mari Deschamps \\ Universidade Federal de Santa Catarina \\ lucianemarideschamps@gmail.com \\ Eduarda Besen \\ Universidade Federal de Santa Catarina \\ dudabesen@gmail.com \\ Emanuelle Moreira \\ Universidade Federal de Santa Catarina \\ emanuelle.ccontato@gmail.com
}

\section{Resumo}

Objetivo: O presente artigo visa descrever o atendimento de crianças com transtornos de sons da fala e delinear sua aplicabilidade por meio da comparação com a literatura. Métodos: O projeto ULTRAFONO consiste em oferecer atendimento às crianças diagnosticadas com transtornos do Som de Fala advindos da Clínica Escola de Fonoaudiologia UFSC, localizada na cidade de Florianópolis (Santa Catarina, Brasil). Fazem parte do grupo avaliado pacientes com alterações de fala e/ou linguagem graves ou ainda com limite terapêutico no tratamento tradicional. Resultados: foram catalogados dois artigos considerados elegíveis para a avaliação da eficácia e aplicabilidade da ultrassonografia de língua como biofeedback no tratamento dos transtornos dos sons da fala. Conclusão:Assim como no projeto ULTRAFONO, a literatura tem mostrado eficácia nos resultados dos pacientes, uma vez que minimiza o tempo de atendimento clínico tradicional.

Palavras-chave: Fonoaudiologia. Fonoterapia. Transtornos da Fala. Ultrassonografia.

\section{TREATMENT CHILDREN WITH SEVERE OR PERSISTENT SPEECH SOUND DISORDERS BY ULTRASOUND TONGUE IMAGING}

Abstract

The article aims to describe the care of children with speech sound disorders and to outline its applicability comparing with the literature. Methods: The ULTRAFONO project consists of offering care to children diagnosed with Speech Sound Disorders arising from the Speech Therapist Clinic - UFSC located in the city of Florianópolis (Santa Catarina, Brazil). The group evaluated includes patients with severe speech and / or language disorders or even with therapeutic limits in traditional treatment. Results: two articles were considered eligible for the evaluation of effectiveness and the applicability of tongue ultrasound as biofeedback in the treatment of speech sound disorders. Conclusion: As in ULTRAFONO, the literature has shown efficacy in the results of patients, since it minimizes the time of clinical care.

Keywords: Speech Therapy. Phonotherapy. Speech Disorders. Ultrasonography.

\section{TRATAMIENTO DE NIÑOS CON ALTERACIONES DEL HABLA GRAVES O PERSISTENTES MEDIANTE ULTRASONOGRAFÍA DEL LENGUA}

\section{Resumen}

Objetivo: El presente artículo tiene como objetivo describir el cuidado de los niños con trastornos del sonido del habla y esbozar su aplicabilidad a través de la comparación con la literatura. Métodos: El proyecto ULTRAFONO ofrece servicio a los niños diagnosticados con trastornos del sonido y del habla de la Escuela Clínica de Terapia del Habla, situado en la ciudad de Florianópolis (Santa Catarina, Brasil). El grupo evaluado incluye pacientes con trastornos severos del habla y/o lenguaje o incluso con límites terapéuticos en el tratamiento tradicional. Resultados: dos artículos se consideraron elegibles para la evaluación de la eficacia y aplicabilidad del ultrasonido de la lengua como biorretroalimentación con ultrasonido en el tratamiento de trastornos del sonido del habla. Conclusión: Al igual que en el proyecto ULTRAFONO, la literatura ha demostrado efectividad en los resultados del paciente, ya que minimiza el tiempo de atención clínica tradicional.

Palavras clave: Logopedia. Fonoterapia. Trastornos del Habla. Ultrasonido de la Lengua. 
Tratamento de crianças com transtornos dos sons da fala graves ou persistentes por meio da ultrassonografia de língua

\section{INTRODUÇÃO}

As crianças com transtornos dos sons da fala (TSF) apresentam inteligibilidade da fala prejudicadas em decorrência das dificuldades de percepção, de produção motora e/ou a representação fonológica dos segmentos da fala e dos aspectos prosódicos (SHRIBERG et al., 2019; NAMASIVAYAM et al., 2020). Os TSF são subdivididos em três grandes subgrupos: atraso de fala; os erros (residuais) de fala e transtornos motores da fala.

Estudos têm mostrado (BACSFALVI \& BERNHARDT, 2011; PRESTON, BRICK, LANDI, 2013; PRESTON et al., 2014, 2017; PRESTON et al., 2016) que o biofeedbackvisual, por meio da USG, durante a terapia de fala, tem alcançado melhores prognósticos terapêuticos (CLELAND, SCOBBIE, WRENCH, 2015).

Durante o tratamento de crianças com TSF, terapeutas realizam com os pacientes o feedback visual para facilitar a produção dos sons alvos trabalhados em terapia. Tradicionalmente, o recurso comumente utilizado para o feedback é o espelho. O espelho permite a visualização dos gestos de lábios e de língua pelos pacientes. No entanto, uma variedade de tecnologias, como acústica e eletropalatografia (BERNHARDT et al., 2003), também podem fornecer feedback espectral ou fisiológico para auxiliar pacientes a entender e observar seus gestos na língua e posições de contato (LEE et al. 2015).

Dentre as metodologias de biofeedback, a ultrassonografia de língua (USG) tem apresentado evidências de melhor custo/benefício. Destaca-se por não ser um procedimento invasivo e apresentar mínima interferência nos movimentos intraorais, além de proporcionar a visualização de imagens obtidas com maior clareza, pois é possível visualizar aspectos tanto estáticos quanto dinâmicos da língua durante a produção motora da fala (WIETHAN et al., 2015; MELO et al., 2016; CLELAND et al., 2015), contribuindo, assim, para o diagnóstico e/ou tratamento do distúrbio.

A técnica consiste em colocar a sonda do aparelho de ultrassom debaixo do queixo, permitindo feedback visual em tempo real da maior parte da superfície da língua em qualquer plano médio-sagital ou coronal. Em ambas as exibições, essa área é limitada pelas sombras do osso, com a ponta da língua em particular, sendo suscetível a uma sombra da mandíbula (CLELAND et al., 2015). Desta forma, o biofeedback na terapia fornece ao terapeuta, paciente e seus familiares um conhecimento adicional sobre a configuração da língua durante a produção de fala, por se tratar de um aparato instrumental para análise e interpretação mais apurados e fidedignos de dados típicos e atípicos da fala. Portanto, é uma ferramenta visual prática e não invasiva (BARBERENA et al., 2014, CLELAND et al., 2015). 
Tratamento de crianças com transtornos dos sons da fala graves ou persistentes por meio da ultrassonografia de língua

$\mathrm{Na}$ clínica fonoaudiológica, o emprego de protocolos validados e observações subjetivas são, geralmente, fatores que definem as diferentes etapas e procedimentos da fonoterapia. Porém, é importante ressaltar que o uso específico de imagens de ultrassom como ferramenta de análise complementar para diagnosticar os TSF incidem em um tópico recente discutido na literatura (LEE, et al. 2015; PRESTON, et al. 2017). Portanto, a contribuição deste aparato instrumental tem propiciado uma interpretação mais apurada e fidedigna de dados típicos e atípicos da fala (BARBERENA et al., 2014; WIETHAN et al., 2015).

Importante salientar o quão necessário se faz a utilização de diversos instrumentos para a análise objetiva da fala e da voz em exames como os citados, por exemplo, a ultrassonografia, visando obter sucesso na terapia fonoaudiológica. Segundo Wertzner et al. (2014), a utilização das imagens de USG, na análise dos movimentos da língua durante a produção de sons, permite visualizar tanto os movimentos quanto os contornos da língua desde a ponta até a raiz, em tempo real. Por isso, cabe ressaltar que este instrumento pode ser útil como biofeedback durante a terapia, fornecendo ao terapeuta, ao paciente e seus familiares um conhecimento adicional sobre as formas da língua se movimentar ao tentar produzir sons.

A ultrassonografia, portanto, apresenta-se como um diferencial para a realização de um diagnóstico mais apurado e fidedigno, facilitando a compreensão e a interpretação do problema por todos os envolvidos e visando uma intervenção mais assertiva e eficiente (CLELAND et al., 2015; PRESTON et al., 2013, PRESTON et al., 2017b). Diante do exposto, faz-se necessário descrever o projeto ULTRAFONO e analisar sua aplicabilidade por meio da comparação deste com a literatura atual.

\section{MATERIAIS E MÉTODOS}

Foi realizada uma busca nas bases científicas Medline (Pubmed), LILACS, SciELO, Cochrane Library e Scopus, eleitos por assunto, sem restrição de tempo e de idioma. Considerando a aplicabilidade dos artigos admitidos neste estudo, os mesmos foram utilizados como base para a organização e encaminhamentos do projeto ULTRAFONO que visa uma estruturação eficiente e de caráter pioneiro.

Os descritores foram selecionados a partir dos vocabulários controlados Descritores em Ciências da Saúde (DeCS) e Medical SubjectHeadingTerms (MeSH), haja vista a sua ampla utilização pela comunidade científica para a indexação de artigos na base de dados PubMed. 
Tratamento de crianças com transtornos dos sons da fala graves ou persistentes por meio da ultrassonografia de língua

Em seguida, foi feita a análise descritiva de dois artigos científicos publicados em bases de saúde, os quais foram avaliados no quesito qualidade pela a escala de Pithon et al. (2015) e que apresentavam no seu escopo o uso da ultrassonografia.

O Projeto ULTRAFONO ocorreu na Clínica Escola de Fonoaudiologia, na UFSC, no período de 2018 a 2020. Os participantes foram crianças (que apresentam cognitivas) que não responderam à terapia fonoaudiológica tradicional para os TSF. As sessões foram individuais e ocorreram uma vez por semana, com duração de 60 minutos cada, sendo os 30 minutos iniciais para a visualização dos movimentos por USF, e 30 minutos finais para a terapia tradicional com atividades envolvendo o mesmo som alvo. A intervenção com o uso da USF foi realizada em momentos distintos, para que os pacientes visualizassem os movimentos da própria língua. No Projeto ULTRAFONO, as avaliações aconteceram sempre que a criança produzia o som que estava sendo trabalhado.

Foram extraídas informações dos artigos avaliados e construídos dois quadros (Quadro 1 e Quadro 2) para a visualização da dinâmica de utilização da ultrassonografia como biofeedback aplicada especificamente a transtornos dos sons da fala (TSF). As variáveis aferidas consistiram em ponderar, principalmente, sobre o modelo ou descrição de intervenção e a alteração fonoaudiológica aplicada. Nesta avaliação, foi possível observar o tempo e a quantidade de sessões de terapia, estímulos trabalhados, além da eficácia dos resultados após intervenção (Quadro 2).

Os estudos selecionados para esta pesquisa indicaram a possibilidade ou não da USF estar associada à terapia tradicional, assim como a quantidade total de sessões; se houve a avaliação pré e pós terapia; o som-alvo a ser trabalhado e quais os resultados e a eficácia pós-terapia.

\section{RESULTADOS E ANÁLISES}

Os dois artigos (PRESTON et al., 2016; CLELAND et al., 2015) que foram selecionados como base teórica do projeto ULTRAFONO propõem o biofeedback durante a terapia, utilizando como instrumento de análise a visualização por imagem de USG das alterações de fala/linguagem. O Quadro 1, a seguir, apresenta, resumidamente, o ano de publicação, objetivos, resultados e conclusões encontrados em cada um dos estudos. 
Tratamento de crianças com transtornos dos sons da fala graves ou persistentes por meio da ultrassonografia de língua

Quadro 1 - Resumo dos objetivos, participantes, resultados e conclusão dos artigos selecionados.

\begin{tabular}{|c|c|c|c|c|}
\hline Autor e ano & Objetivo & Participantes & Resultados & Conclusão \\
\hline $\begin{array}{c}\text { Preston et al., } \\
\text { (2016) }\end{array}$ & $\begin{array}{c}\text { Avaliar o } \\
\text { desempenho } \\
\text { de crianças } \\
\text { durante a } \\
\text { aquisição e } \\
\text { generalização } \\
\text { de róticos do } \\
\text { inglês } \\
\text { americano, } \\
\text { usando feedback } \\
\text { de } \\
\text { ultrassonografi } \\
\text { a de língua. }\end{array}$ & $\begin{array}{l}\text { Três meninos } \\
\text { Faixa etária: } \\
\text { 10,0 a } 13,0 \\
\text { anos de idade }\end{array}$ & $\begin{array}{c}\text { Dois dos } \\
\text { participantes } \\
\text { aumentaram a } \\
\text { precisão da sua } \\
\text { produção } \\
\text { durante as } \\
\text { sessões clínicas } \\
\text { de tratamento, } \\
\text { mas nenhum } \\
\text { sujeito } \\
\text { demonstrou } \\
\text { generalização } \\
\text { para palavras } \\
\text { não tratadas. } \\
\text { Ao tratar a } \\
\text { produção do } \\
\text { /I/, foi possível } \\
\text { contribuir para } \\
\text { melhorar } \\
\text { ainteligibilidade } \\
\text { de fala. }\end{array}$ & $\begin{array}{l}\text { Apesar do sucesso } \\
\text { do treinamento de } \\
\text { feedback visual de } \\
\text { ultrassom para } \\
\text { algumas crianças } \\
\text { com AFI, o uso } \\
\text { deste instrumento } \\
\text { não é uma } \\
\text { abordagem única, } \\
\text { necessitando de } \\
\text { outras estratégias } \\
\text { linguísticas e } \\
\text { motoras para } \\
\text { facilitar a precisão } \\
\text { do som da fala, } \\
\text { principalmente em } \\
\text { indivíduos } \\
\text { comAFIe com } \\
\text { comprometimentos } \\
\text { concomitantes. }\end{array}$ \\
\hline $\begin{array}{c}\text { Cleland, et al. } \\
\text { (2015) }\end{array}$ & $\begin{array}{c}\text { Avaliar a } \\
\text { eficácia no } \\
\text { tratamento de } \\
\text { crianças com } \\
\text { transtornos } \\
\text { persistentes do } \\
\text { som da fala } \\
\text { que não } \\
\text { respondem à } \\
\text { terapia } \\
\text { tradicional. }\end{array}$ & $\begin{array}{c}\text { Oito crianças } \\
\text { Faixa etária: } 6,0 \\
\text { a 10,1 anos de } \\
\text { idade }\end{array}$ & $\begin{array}{l}\text { Houve aumento } \\
\text { médio no } \\
\text { percentual de } \\
\text { consoantes } \\
\text { corretas de } 95 \% \text {, } \\
\text { após seis } \\
\text { semanas de } \\
\text { terapia e } \\
\text { diferenças na } \\
\text { língua no que se } \\
\text { refere à forma. } \\
\text { Todas as } \\
\text { crianças } \\
\text { mostram } 100 \% \\
\text { de não- } \\
\text { sobreposição. }\end{array}$ & $\begin{array}{l}\text { O estudo evidencia } \\
\text { a utilização de USF } \\
\text { para tratar tipos de } \\
\text { erros previamente } \\
\text { não tratados em } \\
\text { uma abordagem } \\
\text { tradicional de } \\
\text { terapia (sem o uso } \\
\text { da } \\
\text { ultrassonografia). } \\
\text { No entanto, os } \\
\text { mecanismos pelos } \\
\text { quais o USG } \\
\text { aprimora a } \\
\text { aprendizagem } \\
\text { ainda não estão } \\
\text { esclarecidos. }\end{array}$ \\
\hline
\end{tabular}

Legenda: AFI - Apraxia de Fala Infantil; USF - Ultrassonografia

As informações relativas à análise dos estudos, a partir de uma avaliação minuciosa, estão descritas no Quadro 2 a seguir: 
Tratamento de crianças com transtornos dos sons da fala graves ou persistentes por meio da ultrassonografia de língua

Quadro 2 - Associação ou não da USF à terapia tradicional, tempo e número de sessões de terapia, estímulos trabalhados e modelo/descrição de intervenções.

\begin{tabular}{|c|c|c|c|c|c|}
\hline $\begin{array}{l}\text { Autores e } \\
\text { ano de } \\
\text { publicação }\end{array}$ & $\begin{array}{c}\text { Associado } \\
\text { ou não à } \\
\text { terapia } \\
\text { tradiciona } \\
1\end{array}$ & $\begin{array}{l}\text { Número de } \\
\text { sessões e } \\
\text { duração }\end{array}$ & $\begin{array}{l}\text { Sons alvos } \\
\text { trabalhados }\end{array}$ & $\begin{array}{l}\text { Alteração } \\
\text { fonoaudioló } \\
\text { gica dos } \\
\text { participantes }\end{array}$ & $\begin{array}{l}\text { Modelo/descrição } \\
\text { de intervenção }\end{array}$ \\
\hline $\begin{array}{l}\text { Preston et } \\
\text { al., (2016) }\end{array}$ & $\operatorname{Sim}$ & $\begin{array}{c}14 \text { sessões } \\
\text { de uma } \\
\text { hora cada. }\end{array}$ & $\begin{array}{l}\text { Róticos do } \\
\text { inglês } \\
\text { americano } \\
(\text { de sons } / \mathrm{x} / \text { ) }\end{array}$ & $\begin{array}{l}\text { Apraxia de } \\
\text { Fala Infantil } \\
\text { (AFI) }\end{array}$ & $\begin{array}{c}\text { Avaliação } \\
\text { Fonoaudiológica: } \\
\text { (a) Porcentagem } \\
\text { de Consoantes } \\
\text { Corretas (PCC), } \\
\text { (b) Porcentagens } \\
\text { de Róticos } \\
\text { Corretos (PRC) e } \\
\text { (c) tarefas diversas } \\
\text { com sílabas, } \\
\text { palavras e } \\
\text { sentenças com os } \\
\text { róticos. } \\
\text { Terapia } \\
\text { fonoaudiológica: } \\
\text { Aprendizagem } \\
\text { motora e a } \\
\text { imagens de USG. }\end{array}$ \\
\hline $\begin{array}{c}\text { Cleland, et al. } \\
\text { (2015) }\end{array}$ & Sim & $\begin{array}{c}12 \text { sessões } \\
\text { (total de } \\
\text { seis } \\
\text { semanas) } \\
\text { O tempo } \\
\text { de cada } \\
\text { sessão de } \\
\text { fonoterapi } \\
\text { a variou } \\
\text { entre } 10 \text { a } \\
50 \\
\text { minutos. }\end{array}$ & $\begin{array}{l}\text { Róticos de } \\
\text { inglês } \\
\text { americano }\end{array}$ & $\begin{array}{c}\text { Erro Residual } \\
\text { de Fala } \\
\text { (ERF) }\end{array}$ & $\begin{array}{c}\text { Avaliações pré e } \\
\text { pós-terapia } \\
\text { (PPC). Terapia } \\
\text { fonoaudiológica: } \\
\text { (pares mínimos, } \\
\text { discriminação } \\
\text { auditiva, } \\
\text { consciência } \\
\text { fonológica e } \\
\text { imagens de USG } \\
\text { para a análise } \\
\text { visual dos } \\
\text { movimentos da } \\
\text { língua durante a } \\
\text { produção dos } \\
\text { róticos). }\end{array}$ \\
\hline
\end{tabular}

A pesquisa de Preston et al. (2016) objetivou a avaliação do desempenho de três meninos com idades entre 10 e 13 anos com erros de fala persistentes associados à AFI, monolíngues de língua inglesa e encaminhados por fonoaudiólogos locais. Os participantes passaram por triagem auditiva e visual. Apresentaram erros persistentes em /I/ nas posições de início e de coda silábica. 
Tratamento de crianças com transtornos dos sons da fala graves ou persistentes por meio da ultrassonografia de língua

O diagnóstico dos participantes foi relatado pelos médicos responsáveis e foi confirmado de forma independente por dois fonoaudiólogos certificados na equipe de pesquisa. A terapia abordou, principalmente, a produção da consoante $/ \mathrm{r} /$, usando a ultrassonografia como técnica de biofeedback visual. Os resultados alcançados foram positivos, visto que a maioria dos participantes obteve aumento de 39\% na porcentagem correta de consoante, na primeira condição tratada, e de $32 \%$, no segmento-alvo em frases e palavras não tratadas.

Cleland et al. (2015) avaliaram a eficácia da técnica de biofeedback com o uso da USG no tratamento de oito crianças entre 6 e 10 anos e 1 mês, falantes monolíngues do inglês, com erros residuais de fala (ERF), que não respondiam à terapia tradicional. O estudo apresentou resultados positivos, porém, limitados. Após a intervenção, todos os participantes realizaram progressos significativos nos segmentos-alvo, no caso, a generalização dos róticos, evidenciados tanto por medidas perceptuais quanto por mudanças na forma da língua. Com relação aos sujeitos participantes dos estudos selecionados, todos apresentaram diagnóstico de um distúrbio relacionado à fala/linguagem, com dificuldades que deveriam ter sido superadas e para as quais o tratamento com terapias fonoaudiológicas convencionais não obtiveram respostas. Os critérios de inclusão proposto por Cleland et al. (2015) também foram consideradas para a seleção das crianças que participaram do Projeto ULTRAFONO.

Em ambos os estudos e no Projeto ULTRAFONO, os pacientes tiveram a oportunidade de visualizar os movimentos de língua em algum momento da terapia. A visualização por imagens de USG, nos estudos, ocorreu em momentos diferentes: antes, durante e após as sessões terapêuticas. Quando os pacientes podiam visualizar os movimentos de língua na produção dos sons que apresentavam dificuldades, comparando-os com o movimento padrão, foi possível a correção dos próprios movimentos durante a produção dos segmentos-alvo.

A Figura 1 demonstra todo o aparato necessário para a captura da imagem da língua durante o processo de avaliação. 
Tratamento de crianças com transtornos dos sons da fala graves ou persistentes por meio da ultrassonografia de língua

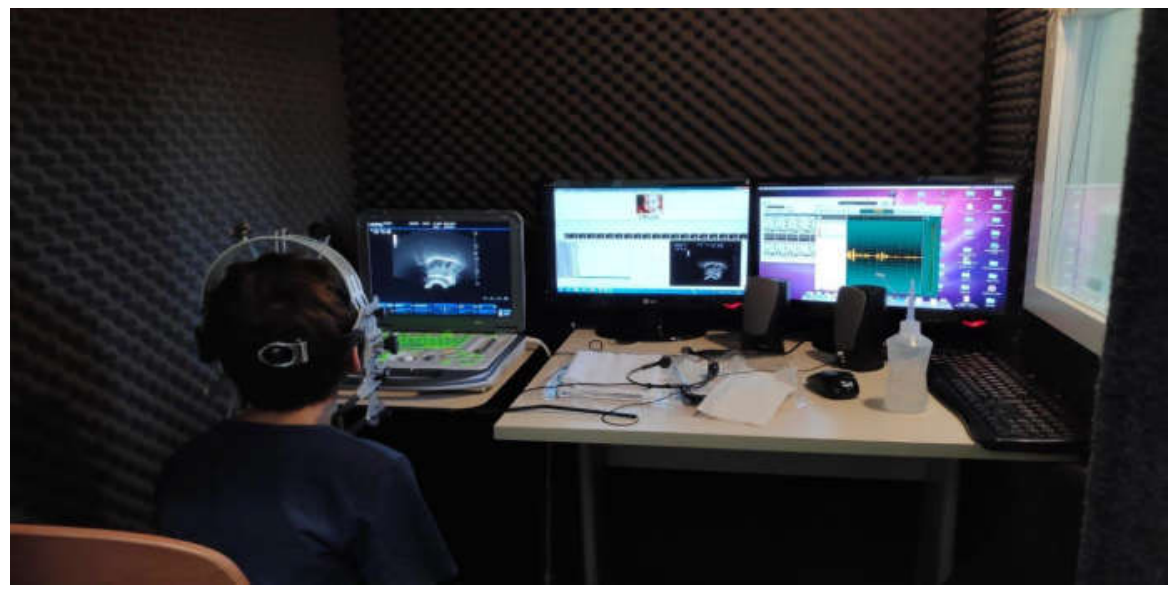

Figura 1: Disposição dos equipamentos utilizados: (1) aparelho de ultrassom, (2) computador com o software, (3) computador para a visualização da criança e do terapeuta, (3) microfone unidirecional e (4) estabilizador de cabeça utilizado nas avaliações.

Fonte: Própria

Importante destacar também que, devido ao conhecimento explícito do próprio desempenho no que se refere à produção de fala, os pacientes podiam empregar os princípios da aprendizagem motora em contextos mais complexos, no qual o som aparecia, generalizando-o. $\mathrm{O}$ biofeedback visual, ao mostrar os movimentos de língua em tempo real, ofereceu às crianças algo novo, um momento de grande avanço na aquisição de novas articulações quando outros tratamentos falharam, fornecendo conhecimento explícito de desempenho. Além disso, ao visualizarem os movimentos de língua em imagens de USG, foi possível aumentar a percepção dos próprios movimentos, visto que o paciente via além dos movimentos dos lábios.

Quanto ao método das terapias com o uso das imagens de USG, no estudo de Cleland et al. (2015), observou-se que o número de sessões para intervenção ocorreu, para cada participante, em um único bloco de 12 sessões de terapia individualizada, com 60 minutos de duração cada, incluindo desde a chamada telefônica para a família, as sessões de ultrassom, até outras atividades fonoaudiológicas aplicadas. O tempo de cada sessão de fonoterapia variou entre 10 a 50 minutos, dependendo da tolerância do participante ao fone, ao instrumento de ultrassom e aos testes avaliativos.

No estudo de Cleland et al. (2015), os sessenta minutos da sessão de terapia foram divididos em dois momentos: durante os 30 minutos iniciais, fez-se o uso das imagens de USG para que o paciente pudesse associar o movimento da imagem na tela do ultrassom com o movimento da própria língua e, também, para que pudesse observara imagem de ultrassom dos movimentos de língua de outras crianças com a mesma idade e sexo, em câmara lenta e frame-por-frame, como modelo visual da articulação dos sons alvos. Esta estratégia possibilitou aos participantes do estudo 
Tratamento de crianças com transtornos dos sons da fala graves ou persistentes por meio da ultrassonografia de língua

experimentarem diferentes variantes fonéticas como alvos e verem línguas de tamanhos similares às suas. Nos 30 minutos finais da terapia, o trabalho terapêutico ocorreu sem o uso do ultrassom e com atividades fonoaudiológicas envolvendo o som alvo trabalhado. No projeto ULTRAFONO, a estrutura empregada para cada sessão também foi a mesma.

Assim como no estudo de Cleland et al. (2015), no projeto ULTRAFONO, cada participante também recebeu atividades fonoaudiológicas individualizadas para casa, durante cinco dias por semana, com o propósito de reforçar os exercícios fonoaudiológicos dados na clínica, mas estes não foram cumpridos integralmente pelas famílias envolvidas devido a outros compromissos com os quais as mesmas se justificaram. As sessões com o instrumento de ultrassom foram restritas a um máximo de 50 min e removidas mais cedo, caso o participante pedisse, por ser este desconfortável e pesado. No entanto, todas as crianças toleraram o fone de ouvido durante todas as sessões de gravação, sem precisar removê-lo ou recolocá-lo. As imagens de USG foram usadas antes, durante e depois da terapia. Durante a terapia, foi conveniente o uso do instrumento para fornecer feedback visual em tempo real e evitar a situação onde a imagem do ultrassom estaria sujeita à movimentação excessiva durante a sessão.

No estudo de Preston et al. (2016), o procedimento das terapias, em cada sessão de tratamento, incluiu a prática com e sem o feedback de ultrassom. $\mathrm{O}$ tratamento neste estudo aconteceu em duas fases, sendo a Fase 1 composta pelas sessões de 1 a 7 , nas quais o objetivo consistia em trabalhar os róticos. Nesta etapa, houve apenas o monitoramento e registro das respostas das crianças. A Fase II, composta pelas sessões de 8 a 14, o tratamento incluiu o uso ou não das pistas prosódicas (melodia da fala) na produção dos róticos.

No projeto ULTRAFONO, os participantes foram atendidos uma vez por semana, individualmente, durante 50 minutos. No período de férias acadêmicas, os discentes participantes do projeto realizaram intervenções intensivas, isto é, de 3 a 5 vezes por semana, durante 50 minutos. Apesar de se considerar o atendimento intensivo mais eficaz para esses casos, por falta de disponibilidade dos discentes e docentes envolvidos, não foi possível a realização das atividades do projeto durante o período concomitante às aulas acadêmicas. Como a avaliação da fala/linguagem dos participantes antes e após as sessões de terapia para fins comparativos foi necessária nos estudos que serviram de base teórica, o Projeto ULTRAFONO também fez uso desta mesma estratégia, avaliando os participantes sempre que estes produziam o som-alvo trabalhado.

Os resultados obtidos no estudo de Cleland et al. (2015), com o uso da USF, apontaram um aumento da acurácia dos fonemas trabalhados após seis semanas de terapia. Todas as crianças participantes do estudo mostraram 100\% de não-sobreposição e ainda foi possível identificar significativa diferença entre pré/pós-terapia no que se refere ao contorno da língua. 
Tratamento de crianças com transtornos dos sons da fala graves ou persistentes por meio da ultrassonografia de língua

Quanto aos resultados das intervenções no estudo de Preston et al. (2015), que tratou crianças com AFI, observou-se que dois dos participantes aumentaram a precisão da sua produção durante as sessões de tratamento. Os autores perceberam que tanto o desempenho quanto o aprendizado dos participantes foram significativos após a terapia com o uso do feedback visual. Cabe esclarecer que o desempenho se refere à precisão em uma tarefa durante o treinamento para a aquisição do som-alvo, enquanto o aprendizado requer generalização para estímulos não treinados, bem como retenção deste mesmo estímulo ao longo do tempo.

\section{CONSIDERAÇÕES FINAIS}

Os dois estudos selecionados como base teórica, assim como o próprio projeto ULTRAFONO, concluíram que o uso do instrumento de ultrassonografia de língua concomitantemente à terapia convencional podem efetivar a superação dos erros de fala oriundos dos transtornos dos sons da fala. No entanto, os autores concordam que há necessidade de novas pesquisas neste âmbito, visto que estudos envolvendo o uso de tecnologias na área da fala ainda são inovadores no Brasil.

Apesar das vantagens já mencionadas com relação a outras técnicas instrumentais (McLEOD e SEARl, 2006; KATZ W, et al. 2014), a USG de língua apresenta limitações, ou seja, a impossibilidade de se visualizar estruturas, como o palato duro, o palato mole e as paredes da faringe e a qualidade da imagem do contorno da língua, também pode se mostrar comprometida. Outra limitação diz respeito ao custo dos equipamentos envolvidos que, para um terapeuta, pode dificultar o acesso e reforçar a implementação de equipamentos de alta tecnologia no atendimento à comunidade.

A limitação observada durante a execução do projeto ULTRAFONO foi a impossibilidade de ampliar o tratamento para um número maior de crianças com transtornos dos sons de fala, já que o uso do equipamento permanece restrito a um único paciente de cada vez.

Os dados sugerem que a USG pode ser altamente eficaz em tratar os erros de fala não tratados na terapia tradicional. Também permite aos pacientes o conhecimento explícito sobre a sua produção de fala, pois estes podem empregar os princípios da aprendizagem motora, principalmente em contextos mais complexos em que o som aparece, estabelecendo generalizações para sons não tratados. Portanto, o biofeedback visual tem caráter inovador, um grande avanço na aquisição de sons quando a criança apresenta dificuldades de produção, bem como quando outros tratamentos tradicionais falharem ou apresentarem poucos sucessos terapêuticos. 
Tratamento de crianças com transtornos dos sons da fala graves ou persistentes por meio da ultrassonografia de língua

\section{REFERÊNCIAS}

ASHA. Speech Sound Disorders-Articulation and Phonology. 2007. Disponível em: https://www.asha.org/practice-portal/clinical-topics/articulation-and-phonology/.

BACSFALVI, Penelope; BERNHARDT, Barbara May. Long-term outcomes of speech therapy for seven adolescents with visual feedback technologies: ultrasound and electropalatography. Clinical Linguistics \& Phonetics, [S.L.], v. 25, n. 11-12, p. 1034-1043, nov. 2011. Informa UK Limited. http://dx.doi.org/10.3109/02699206.2011.618236.

BARBERENA, Luciana da Silva; BRASIL, Brunah de Castro; MELO, Roberta Michelon; MEZZOMO, Carolina Lisbôa; MOTA, Helena Bolli; KESKE-SOARES, Márcia. Ultrasound applicability in Speech Language Pathology and Audiology. Codas, [S.L.], v. 26, n. 6, p. 520530, dez. 2014. FapUNIFESP (SciELO). http://dx.doi.org/10.1590/2317-1782/20142013086.

BARLOW, David H.; HERSEN, Michel. Single case experimental designs: strategies for studying behavior change. 2. ed. New York: Pergamon Press, 1982.

BERNHARDT, Bárbara et al. Habilitação da fala de adolescentes com deficiência auditiva por meio de eletropalatografia e ultrassom avaliada por ouvintes treinados. Clinical Linguistics \& Phonetics, v. 17, n. 3, pág. 199-216, 2003.

BERTI, Larissa Cristina; PAGLIUSO, Alessandra; LACAVA, Francine. Instrumento de avaliação de fala para análise acústica (IAFAC) baseado em critérios linguísticos. Revista da Sociedade Brasileira de Fonoaudiologia, [S.L.], v. 14, n. 3, p. 305-314, 2009. FapUNIFESP (SciELO). http://dx.doi.org/10.1590/s1516-80342009000300005.

BRASIL, Brunah de Castro; MEZZOMO, Carolina Lisbôa; MOTA, Helena Bolli; MELO, Roberta Michelon; LOVATTO, Liane; ARZENO, Leonardo. Características acústicas dos fones [s] e [〕] de adultos e crianças com desenvolvimento fonológico típico. Revista da Sociedade Brasileira de Fonoaudiologia, [S.L.], v. 17, n. 2, p. 182-188, jun. 2012. FapUNIFESP (SciELO). http://dx.doi.org/10.1590/s1516-80342012000200014.

CERON, Marizete Ilha. Instrumento de Avaliação Fonológica (INFONO): Desenvolvimento e estudos psicométricos. 2015. 148 f. Tese (Doutorado) - Curso de Curso de Doutorado do Programa de Pós-Graduação em Distúrbios da Comunicação Humana, Ccs, Universidade Federal de Santa Maria, Santa Maria, 2015.

CIELO, Carla Aparecida; CASARIN, Maísa Tatiana. Sons fricativos surdos. Revista Cefac, [S.L.], v. 10, n. 3, p. 352-358, 2008. Fap UNIFESP (SciELO). http://dx.doi.org/10.1590/s151618462008000300010 .

CLELAND, Joanne; SCOBBIE, James M.; ROXBURGH, Zoe; HEYDE, Cornelia; WRENCH, Alan. Enabling New Articulatory Gestures in Children With Persistent Speech Sound Disorders Using Ultrasound Visual Biofeedback. Journal Of Speech, Language, And Hearing Research, [S.L.], v. 62, n. 2, p. 229-246, 26 fev. 2019. American Speech Language Hearing Association. http://dx.doi.org/10.1044/2018_jslhr-s-17-0360.

CLELAND, Joanne; SCOBBIE, James M.; WRENCH, Alan A.. Using ultrasound visual biofeedback to treat persistent primary speech sound disorders. Clinical Linguistics \& 
Tratamento de crianças com transtornos dos sons da fala graves ou persistentes por meio da ultrassonografia de língua

Phonetics, [S.L.], v. 29, n. 8-10, p. 575-597, 9 mar. 2015. Informa UK Limited. http://dx.doi.org/10.3109/02699206.2015.1016188.

DODD, B. et al. Diagnóstico diferencial de desvios fonológicos. In.: B. Dodd (Ed.). Diagnóstico diferencial e tratamento de crianças com distúrbio da fala/Diferencial Diagnosis And Treatment of Children with Speech Disorder. Second Edition. London and Philadelphia: WhurrPublishers, pp. 44-70. 2005.

FERRANTE, Carla; VAN BORSEL, John; PEREIRA, Mônica Medeiros de Britto. Aquisição fonológica de crianças de classe socioeconômica alta. Revista Cefac, [S.L.], v. 10, n. 4, p. 452 460, dez. 2008. FapUNIFESP (SciELO). http://dx.doi.org/10.1590/s1516-18462008000400005.

FRANCISCO, Danira Tavares; WERTZNER, HaydéeFiszbein. Differences between the production of [s] and [J] in the speech of adults, typically developing children, and children with speech sound disorders: an ultrasound study. Clinical Linguistics \& Phonetics, [S.L.], v. 31, n. 5, p. 375-390, 13 jan. 2017. Informa UK Limited. http://dx.doi.org/10.1080/02699206.2016.1269204.

GIBSON, Tahira; LEE, Sue Ann S. Use of ultrasound visual feedback in speech intervention for children with cochlear implants. ClinicalLinguistics\&Phonetics, [S.L.], p. 1-20, 17 jul. 2020. Informa UK Limited. http://dx.doi.org/10.1080/02699206.2020.1792996.

KATZ William F. et al. Opti-speech: A real-time, 3D visual feedback system for speech training. Proc. Interspeech. p. 1174-1178. Singapore. 14-18 Sep. 2014.

LAMPRECHT, Regina Ritter. Sobre os desvios fonológicos. In: LAMPRECHT, Regina Ritter. (Ed.). Aquisição Fonológica do Português: Perfil de desenvolvimento e subsídios para a terapia. 1. Porto Alegre: ArtmedEditora, v.1, cap. 12, p.193-212. 2004.

LAW, James. Prevalence and natural history of primary speech and language delay: findings from a systematic review of the literature. International Journal Of Language \& Communication Disorders, [S.L.], v. $35, \quad$ n. 2 , p. 165-188, 1 abr. 2000. Wiley. http://dx.doi.org/10.1080/136828200247133.

LAZZAROTTOO-VOLCÃO, Cristiane. Modelo padrão de aquisição de contrastes: uma proposta de avaliação e classificação dos desvios fonológicos. Tese de Doutorado. Programa de Pós-Graduação em Letras. Universidade Católica de Pelotas, Pelotas. 2009.

LEE, Sue Ann S.; WRENCH, Alan; SANCIBRIAN, Sherry. How To Get Started With Ultrasound Technology for Treatment of Speech Sound Disorders. Perspectives On Speech Science And Orofacial Disorders, [S.L.], v. 25, n. 2, p. 66-80, out. 2015. American Speech Language Hearing Association. http://dx.doi.org/10.1044/ssod25.2.66.

LIMA, Fernanda Leitão de Castro Nunes de; SILVA, Cássio Eduardo Esperandino da; SILVA, Lídia Maurício da; VASSOLER, Aline Mara de Oliveira; FABRON, Eliana Maria Gradim; BERTI, Larissa Cristina. Ultrasonographic analysis of lateral liquids and coronal fricatives:

judgment of experienced and non-experienced judges. Revista Cefac, [S.L.], v. 20, n. 4, p. 422431, ago. 2018. FapUNIFESP (SciELO). http://dx.doi.org/10.1590/1982-0216201820412317.

MCLEOD, Sharynne; SEARL, Jeff. Adaptation to an Electropalatograph Palate: acoustic, impressionistic, and perceptual data. American Journal Of Speech-Language Pathology, [S.L.], 
Tratamento de crianças com transtornos dos sons da fala graves ou persistentes por meio da ultrassonografia de língua

v. 15, n. 2, p. 192-206, maio 2006. American Speech LanguageHearingAssociation. http://dx.doi.org/10.1044/1058-0360(2006/018).

MELO, Roberta Michelon; DIAS, Roberta Freitas; MOTA, Helena Bolli; MEZZOMO, Carolina Lisbôa. Imagens de ultrassonografia de língua pré e pós terapia de fala. Revista Cefac, [S.L.], v. 18, n. 1, p. 286-297, fev. 2016. FapUNIFESP (SciELO). http://dx.doi.org/10.1590/19820216201618114515 .

MORGAN, Angela T; MURRAY, Elizabeth; LIÉGEOIS, Frederique J. Interventions for childhood apraxia of speech. Cochrane Database of Systematic Reviews 2018, Issue 5, Art.No.:CD006278 Wiley \& Sons Ltd. http://dx.doi.org/10.1002/14651858.cd006278.pub3.

NAMASIVAYAM, Aravind K.; PUKONEN, Margit; GOSHULAK, Debra; GRANATA, Francesca; LE, D. James; KROLL, Robert; LIESHOUT, Pascal. Investigating intervention dose frequency for children with speech sound disorders and motor speech involvement. International Journal Of Language \& Communication Disorders, [S.L.], p. 673-686, 2 abr. 2019. Wiley. http://dx.doi.org/10.1111/1460-6984.12472.

NEWMEYER, Amy J.; GRETHER, Sandra; GRASHA, Carol; WHITE, Jaye; AKERS, Rachel; AYLWARD, Christa; ISHIKAWA, Keiko; DEGRAUW, Ton. Fine Motor Function and OralMotor Imitation Skills in Preschool-Age Children With Speech-Sound Disorders. Clinical Pediatrics, [S.L.], v. 46, n. 7, p. 604-611, 14 maio 2007. SAGE Publications. http://dx.doi.org/10.1177/0009922807299545.

PRESTON, Jonathan L.; BRICK, Nickole; LANDI, Nicole. Ultrasound Biofeedback Treatment for Persisting Childhood Apraxia of Speech. American Journal Of SpeechLanguage Pathology, [S.L.], v. 22, n. 4, p. 627-643, nov. 2013. American Speech LanguageHearingAssociation. http://dx.doi.org/10.1044/1058-0360(2013/12-0139).

PRESTON, Jonathan L.; MCCABE, Patricia; RIVERA-CAMPOS, Ahmed; WHITTLE, Jessica L.; LANDRY, Erik; MAAS, Edwin. Ultrasound Visual Feedback Treatment and Practice Variability for Residual Speech Sound Errors. Journal Of Speech, Language, And Hearing Research, [S.L.], v. 57, n. 6, p. 2102-2115, dez. 2014. American Speech Language Hearing Association. http://dx.doi.org/10.1044/2014_jslhr-s-14-0031.

PRESTON, Jonathan L.; LEECE, Megan C.; MAAS, Edwin. Intensive Treatment with Ultrasound Visual Feedback for Speech Sound Errors in Childhood Apraxia. Frontiers In Human Neuroscience, [S.L.], v. 10, 30 ago. 2016. Frontiers Media SA. http://dx.doi.org/10.3389/fnhum.2016.00440.

PRESTON, Jonathan L.; LEECE, Megan C.; MAAS, Edwin. Motor-based treatment with and without ultrasound feedback for residual speech-sound errors. International Journal Of Language \& Communication Disorders, [S.L.], v. 52, n. 1, p. 80-94, 14 jun. 2016. Wiley. http://dx.doi.org/10.1111/1460-6984.12259.

PRESTON, Jonathan L.; BYUN, Tara McAllister; BOYCE, Suzanne E.; HAMILTON, Sarah; TIEDE, Mark; PHILLIPS, Emily; RIVERA-CAMPOS, Ahmed; WHALEN, Douglas H.. Ultrasound Images of the Tongue: a tutorial for assessment and remediation of speech sound errors. Journal Of Visualized Experiments, [S.L.], n. 119, p. 1-15, 3 jan. 2017. MyJove Corporation. http://dx.doi.org/10.3791/55123. 
Tratamento de crianças com transtornos dos sons da fala graves ou persistentes por meio da ultrassonografia de língua

PRESTON, Jonathan L.; MAAS, Edwin; WHITTLE, Jessica; LEECE, Megan C.; MCCABE, Patricia. Limited acquisition and generalisation of rhotics with ultrasound visual feedback in childhood apraxia. Clinical Linguistics \& Phonetics, [S.L.], v. 30, n. 3-5, p. 363-381, 3 ago. 2015. Informa UK Limited. http://dx.doi.org/10.3109/02699206.2015.1052563.

RABELO, Alessandra Terra Vasconcelos; ALVES, Claudia Regina Lindgren; GOULART, Lúcia Maria H. Figueiredo; FRICHE, Amélia Augusta de Lima; LEMOS, Stela Maris Aguiar; CAMPOS, Fernanda Rodrigues; FRICHE, Clarice Passos. Alterações de fala em escolares na cidade de Belo Horizonte. Jornal da Sociedade Brasileira de Fonoaudiologia, [S.L.], v. 23, n. 4, p. 344-350, dez. 2011. FapUNIFESP (SciELO). http://dx.doi.org/10.1590/s2179-64912011000400009.

RUSSO, Iêda Chaves Pacheco; BEHLAU, Mara. As pistas acústicas das vogais e consoantes. Percepção da fala: análise acústica do português brasileiro. São Paulo: Lovise. 1993.

SCOBBIE, James M. et al. Head-Probe Stabilisation in Ultrasound Tongue Imaging Using a Headset to Permit Natural Head Movement. The 8th International Seminar on Speech Production, Strasbourg, Université Marc Bloch, p. 373-376, 2008.

SHRIBERG, Lawrence D.; AUSTIN, Diane; LEWIS, Barbara A.; MCSWEENY, Jane L.; WILSON, David L.. The Percentage of Consonants Correct (PCC) Metric. Journal Of Speech, Language, And Hearing Research, [S.L.], v. 40, n. 4, p. 708-722, ago. 1997. American Speech Language Hearing Association. http://dx.doi.org/10.1044/jslhr.4004.708.

SHRIBERG, Lawrence D.; POTTER, Nancy L.; STRAND, Edythe A.. Prevalence and Phenotype of Childhood Apraxia of Speech in Youth With Galactosemia. Journal Of Speech, Language, And Hearing Research, [S.L.], v. 54, n. 2, p. 487-519, abr. 2011. American Speech LanguageHearingAssociation. http://dx.doi.org/10.1044/1092-4388(2010/10-0068).

SHRIBERG, Lawrence D.; CAMPBELL, Thomas F.; KARLSSON, Heather B.; BROWN, Roger L.; MCSWEENY, Jane L.; NADLER, Connie J.. A diagnostic marker for childhood apraxia of speech: the lexical stress ratio. Clinical Linguistics \& Phonetics, [S.L.], v. 17, n. 7, p. 549-574, out. 2003. Informa UK Limited. http://dx.doi.org/10.1080/0269920031000138123.

SILVA, Rafaela D. Terapia fonoaudiológica por meio da ultrassonografia no distúrbio do som de fala: relato de caso. TCC (Fonoaudiologia), Universidade Federal de Santa Catarina, Florianópolis (SC), 2020.

STACKHOUSE, Joy. Fala, ortografia e leitura: quem está em risco e porquê. In: Snowling, Margaret Stackhouse, Joy. Dislexia, Fala e Linguagem:Um manual do profissional (M.F. Lopes, Trad.), pp. 23-27. Porto Alegre: Artmed Editora.

SUGDEN, Eleanor; LLOYD, Susan; LAM, Jenny; CLELAND, Joanne. Systematic review of ultrasound visual biofeedback in intervention for speech sound disorders. International Journal Of Language \& Communication Disorders, [S.L.], v. 54, n. 5, p. 705-728, 10 jun. 2019. Wiley. http://dx.doi.org/10.1111/1460-6984.12478.

VASSOLER, Aline Mara de Oliveira; BERTI, Larissa Cristina. Análise ultrassonográfica quantitativa da produção de encontros consonantais realizada por crianças típicas $\mathrm{e}$ atípicas. In: Anais do XXIII Congresso Brasileiro e IX Congresso Internacional de Fonoaudiologia. São Paulo: SBFA, 2015. p. 14-16. 
Tratamento de crianças com transtornos dos sons da fala graves ou persistentes por meio da ultrassonografia de língua

YAVAS, Mehmet; HERNANDORENA, Carmen L; LAMPRECHT, Regina R. Avaliação Fonológica da Criança Reeducação e Terapia. Porto Alegre: Artmed, 2002. 150 p.

WIETHAN, Fernanda; CERON, Marizete Ilha; MARCHETTI, Paula; GIACCHINI, Vanessa; MOTA, Helena Bolli. O uso da eletroglotografia, eletromiografia, espectrografia e ultrassom nos estudos de fala - revisão teórica. Revista Cefac, [S.L.], v. 17, n. 1, p. 115-125, mar. 2015. FapUNIFESP (SciELO). http://dx.doi.org/10.1590/s1516-18462013005000049.

WIETHAN, Fernanda Marafiga; MOTA, Helena Bolli. Emprego de estratégias de reparo para os fonemas fricativos no desvio fonológico. Revista da Sociedade Brasileira de Fonoaudiologia, [S.L.], v. 17, n. 1, p. 28-33, mar. 2012. FapUNIFESP (SciELO). http://dx.doi.org/10.1590/s151680342012000100007.

WERTZNER, Haydée Fiszbein; FRANCISCO, Danira Tavares; PAGAN-NEVES, Luciana de Oliveira. Tongue contour for /s/ and /?/ in children with speech sound disorder. Codas, [S.L.], v. 26, n. 3, p. 248-251, jun. 2014. FapUNIFESP (SciELO). http://dx.doi.org/10.1590/2317$1782 / 201420130022$.

Recebido em: 12/06/2020

Aceito em: 05/04/2021 